\title{
EINIGE BEOBACHTUNGEN AN ANEMONENFISCHEN IN DER KARIBISCHEN SEE
}

\author{
von \\ H. ALBRECHT \\ Abteilung Tierverbalten, Universität von Amsterdam, die Niederlande
}

\begin{abstract}
SUMMARY
Anemone fishes shelter in sea anemones. Well known is the symbiosis of the indopacific genus Ampbiprion (Pomacentridae) with Stoichactis gigantea and some other anemones.

The present paper records some 30 fishes, members of 12 different families, associated with Condylactis gigantea in Curaçao, Caribbean Sea. It concerns mostly juveniles of small, inconspicuous fish species.
\end{abstract}

\section{ZUSAMMENFASSUNG}

Anemonenfische sind Fische, die in Seeanemonen Schutz finden. Bekannt und aufällig sind 26 Amphiprion-Arten aus der Familie der Riffbarsche (Pomacentriden). Ampbiprion kommt in Riffen des Indopazifik vor und ist mit Stoichactis gigantea vergesellschaftet.

Aus Condylactis gigantea von Curaçao, Karibische See, werden etwa 30 Anemonenfischarten aus 12 Familien vorgestellt. Es handelt sich dabei um kleine, unauffällige Arten, vor allem aber um Jungfische.

\section{EINLEITUNG}

Unter Anemonenfischen verstehen wir hauptsächlich Arten der indopazifischen Gattung Amphiprion (Riffbarsche, Pomacentridae), die in Seeanemonen leben. Mariscal (1966) und neuerdings Allen (1975) haben die vielen Untersuchungen dieser Beziehung zusammengefasst. Aus der Literatur sind andere Anemonenfische kaum bekannt: es handelt sich um einen Gobiiden aus dem Mittelmeer (Abel, 1960) und ungefähr 14 Arten aus dem gleichen Lebensraum wie Amphiprion, darunter Pomacentriden (Stevenson, 1963), Cirrhitiden (Eibl-Eibesfeldt, 1964), Labriden, Blenniiden, Apogoniden, Anthiiden (Graefe, 1964). Bei all diesen Arten ist der Kontakt der Fische mit den
Tentakeln der Anemone bei weitem nicht so deutlich ausgeprägt wie bei Amphiprion. Es scheint, dass die schützende Nähe der Tentakeln zwar aufgesucht, direkter Kontakt aber meistens gemieden wird.

Neuerdings werden Anemonenfische auch in der Karibischen See gefunden (siehe Karte). Smith (1973) beschreibt einen Cliniden von $\mathrm{Pa}$ nama. Hannau \& Mock (1974) illustrieren eine zweite Clinidenart von den Bahamas. Colin \& Heiser (1973) beschreiben zwei Apogoniden von Jamaica und Curaçao. Hanlon \& Kaufman (1976) fügen aus den Bahamas, von Jamaica und Honduras sechs weitere Arten hinzu: drei Cliniden, einen Gobiiden, einen Labriden und einen Pomadasyiden. Der vorliegende Bericht beschreibt 31 Anemonenfischarten aus 12 Familien von Curaçao, drei Viertel davon neu in dieser Hinsicht. Der Kontakt mit Anemonententakeln stört all diese Fische nicht. Sie scheinen den Kontakt z.T. aufzusuchen. Ob die Vergesellschaftung eine Symbiose ist, ist noch nicht deutlich; sicher ist die Vergesellschaftung nicht so auffällig wie bei Amphiprion.

\section{DANKSAGUNG}

Die Beobachtung wurde ermöglicht durch ein Stipendium von WOTRO (Stiftung zur Förderung der Erforschung der Tropen), Den Haag. Prof. J. H. Stock, Institut für Taxonomische Zoologie, Universität von Amsterdam, gab mir zwei Monate Arbeitsurlaub. Er entdeckte übrigens u.a. den ersten Anemonenfisch in der karibischen See, da die Beobachtung von Pax (1910) praktisch in Vergessenheit geraten war. Dr. I. Kristensen, CARMABI (Karibisch Marin-biologisches 
TABELLE I

Liste der Anemonenfische in Condylactis gigantea aus Curaçao

\begin{tabular}{|c|c|c|c|c|c|c|c|}
\hline Art & Familie & $\begin{array}{r}\text { Län } \\
\text { min. }\end{array}$ & $\begin{array}{l}\text { in Ane } \\
\text { indardlän } \\
\text { in } \mathrm{mm} \text { ) } \\
\text { durch- } \\
\text { schnitt }\end{array}$ & one & $\begin{array}{l}\text { Länge } \\
\text { Adult }\end{array}$ & $\begin{array}{l}\text { Betäubungs- } \\
\text { experiment }\end{array}$ & $\begin{array}{c}\text { in Sammlung } \\
\text { ZMA }\end{array}$ \\
\hline Myripristis jacobus Cuvier & Holocentridae & & 30 & & 200 & + & \\
\hline Holocentrus spec. & " & & 30 & & 250 & & + \\
\hline Symphurus spec. & Cynoglossidae & & 33 & & $?$ & & + \\
\hline Apogon maculatus (Poey) & Apogonidae & 17 & 23 & 32 & 120 & + & + \\
\hline Apogon spec. & ". & 16 & 20 & 34 & 80 & + & + \\
\hline Cephalopholis fulva (Linnaeus) & Serranidae & & 100 & & 300 & & \\
\hline Chaetodon capistratus (Linnaeus) & Chaetodontidae & & 20 & & 150 & + & \\
\hline Chaetodon striatus Linnaeus & " & & 20 & & 150 & & \\
\hline Centropyge argi Woods \& Kanazawa & $"$ & & 20 & & 60 & & \\
\hline Chromis multilineata (Guichenot) & Pomacentridae & 20 & 22 & 30 & 130 & & \\
\hline Eupomacentrus partitus (Poey) & 川 & 14 & 26 & 43 & 80 & & + \\
\hline Eupomacentrus leucostictus (Müller \& & & & & & & + & + \\
\hline Troschel) & $"$ & & 25 & & 120 & & \\
\hline Amblycirrbitus pinos (Mowbray) & Cirrhitidae & & 30 & & 90 & & \\
\hline Halichoeres bivittatus (Bloch) & Labridae & & 25 & & 200 & & \\
\hline \multicolumn{8}{|l|}{ Halichoeres maculipinna (Müller \& } \\
\hline Troschel) & " & & 25 & & 120 & + & + \\
\hline Halichoeres garnoti (Valenciennes) & $"$ & & 25 & & 200 & & \\
\hline Thalassoma bifasciatum (Bloch) & $"$ & 12 & 31 & 67 & 120 & + & + \\
\hline Hemipteronotus splendens (Castelnau) & $"$ & & 25 & & 120 & + & \\
\hline Hemipteronotus novacula (Linnaeus) & , & & 25 & & 150 & & \\
\hline Scarus vetula Bloch \& Schneider & Scaridae & & 25 & & 600 & & + \\
\hline Scarus spec. & , & & 25 & & 300 & & \\
\hline \multicolumn{8}{|l|}{ Sparisoma chrysopterum (Bloch \& } \\
\hline Schneider) & " & & 25 & & 450 & & \\
\hline Sparisoma viride (Bonnaterre) & $"$ & & 25 & & 600 & & \\
\hline \multicolumn{8}{|l|}{ Hemiemblemaria simulus Longley \& } \\
\hline Hildebrand & Clinidae & & 25 & & 120 & & \\
\hline Malacoctenus spec. & , & & 30 & & $?$ & + & + \\
\hline Malacoctenus spec. & $"$ & & 35 & & $?$ & & + \\
\hline Malacoctenus spec. & $"$ & & 35 & & $?$ & & + \\
\hline Malacoctenus spec. & $"$ & & 30 & & $?$ & & + \\
\hline Coryphopterus glaucofraenum Gill & Gobiidae & 13 & 31 & 46 & 75 & + & + \\
\hline Gnatbolepis thompsoni Jordan & , & 14 & 30 & 39 & 67 & & + \\
\hline Scorpaena plumieri Bloch & Scorpaenidae & 10 & 25 & 35 & 450 & + & + \\
\hline
\end{tabular}

Die Liste ist sicher nicht vollständig, da einige der Artbeschreibungen meiner Helfer auf keine der von mir identifizierten Arten passen und da bis zum 17. (letzten) Beobachtungstag neue Arten in Anemonen gesehen wurden.

Institut), Curaçao und seine Mitarbeiter gewährten mir Unterkunft und anregende Diskussionen. Ihnen allen sowie meinen begeisterten Helfern unter Wasser, M. Butter, E. van Dijk, P. Hoetjes, T. van 't Hof, M. Wapstra und E. Westinga, sei gedankt. Den Herren den Hartog, Kristensen und Stock danke ich für kritische Durchsicht des Manuskriptes.

\section{METHODE}

Die vorliegenden Beobachtungen wurden im November 1975 während 90 Stunden in $2-40 \mathrm{~m}$ Tiefe in Curaçao gemacht. Hilfsmittel waren Pressluftatemgeräte, Plexiglasschreibtafel und Bleistift, Stoppuhr, Fangnetze, Plastikbeutel, Pinzette, Rotenon (Atemgift), Foto- und Filmkamera. Formolexemplare der meisten untersuchten Arten wurden mitgebracht und be- finden sich in der Sammlung des Zoologischen Museums, Amsterdam, unter den Nummern ZMA 114.220-114.260. Sie wurden bestimmt mit Hilfe von „Fishes of the Bahamas and adjacent tropical waters" von Böhlke \& Chaplin (1968).

\section{VERBREITUNG}

Alle genannten Fischarten gehören vor allem zu tropischen Familien, die am Boden, meist in Riffen, leben. Man sieht praktisch nur Jungfische in den Tentakeln. In Curaçao kamen die Fische praktisch rundum die Insel vor und die Anemonen 


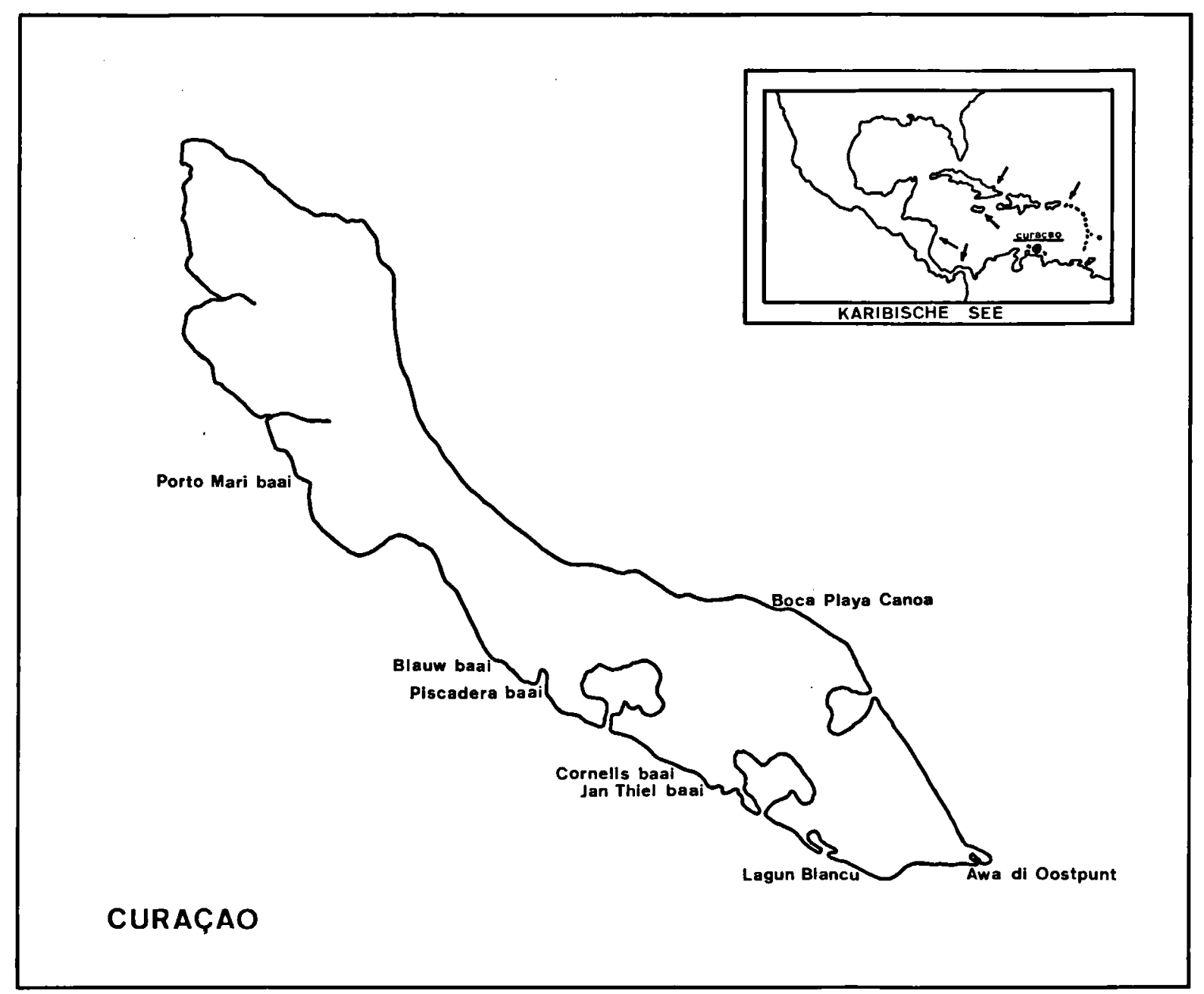

Karte der Karibischen See (rechts oben); Pfeile sind Fundorte von Anemonenfischen. Die Hauptkarte stellt Curaçao dar; die Namen verweisen auf Buchten, in denen nach Anemonenfischen gesucht wurde.

in den meisten Buchten der Südküste (siehe Karte). Es gab einige Stellen, an denen zwar Fische und Anemonen häufig waren, aber kein Fisch (auch kein Jungfisch) in die Tentakeln schwamm. Vielleicht entsteht hier eine Anemonen-Fisch-Vergesellschaftung so wie Graefe (1964) für die Evolution der Amphiprion-Anemonenfisch Symbiose annimmt: ,vermutlich begann es damit, dass kleine und kleinste Jungfische Aktinien besiedelten".

Die Anemone Condylactis ist weltweit in tropischen Meeren verbreitet. Die karibische Condylactis gigantea (Weinland) kommt an der Südküste Curaçaos an einigen Stellen nicht vor, wo das Riff praktisch ungestört und deswegen unzer- stört ist. Sie sind häufig in einer unberührten flachen Lagune (Awa di Oostpunt) und in den Buchten, die vom Menschen stark verändert wurden, wohl am häufigsten an der Pier des Hilton Hotels (Piscadera Baai), wo das Riff durch Spren-

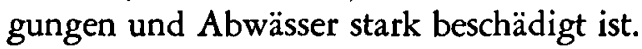

\section{VERHALTEN}

1. Verhalten der Anemone. - Die Tentakeln bleiben bei Berührung durch einen AnemonenFisch entspannt. Gelegentlich sieht man Juvenile Thalassoma bifasciatum und Halichoeres garnoti leicht an Tentakeln zupfen. Die Tentakeln zucken dann örtlich etwas. Nachts werden die Tentakeln dünner und länger, vermutlich zum Planktonfang. 


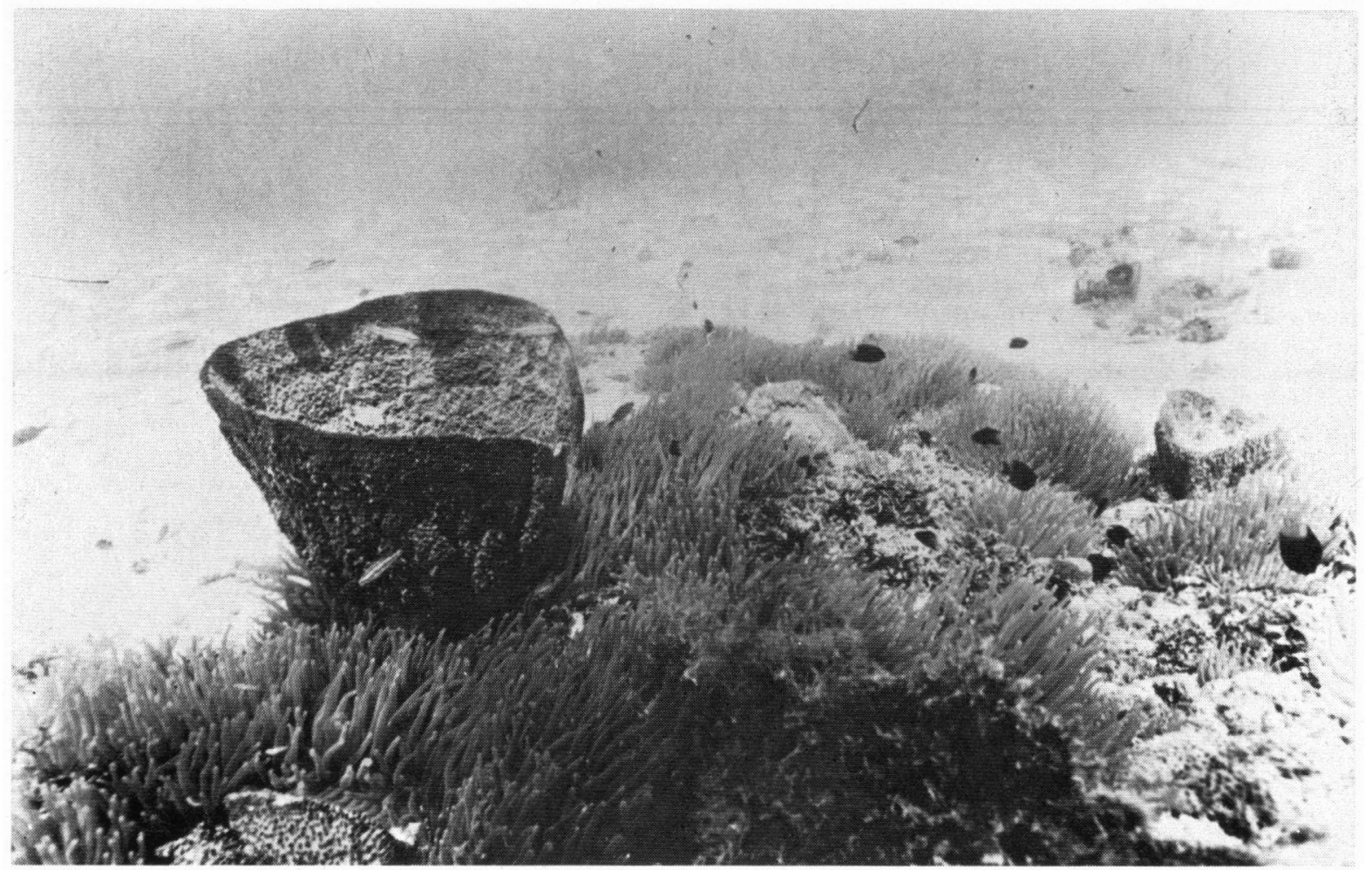

Abb. 1. Auf sandigen Flächen konzentrieren sich viele Fischarten in der Nähe von Deckungsplätzen; hier bei einem ausgedehnten Anemonenrasen.

Die Tentakeln kleben gelegentlich an der Hand des Beobachters, sie verursachen bei Menschen mit empfindlicher Haut rote, juckende Flecken. Frisch geschlüpfte Seeschildkröten (Caretta) werden gefangen und gefressen (de Boer, mdl. Mitt.). Vielleicht fressen sie auch Fische.

2. Verhalten der Fische. - Nur einen adulten Thalassoma bifasciatum (ca. $10 \mathrm{~cm}$ ) sah man in den Tentakeln sich „kuscheln” ähnlich wie es von Amphiprion bekannt ist. Bei allen anderen Individuen und Arten aus Tab. I sah man kein besonderes Verhalten gegenüber der Anemone und auch nicht von der Anemone gegenüber den Fischen. Hanlon \& Kaufman (1976) beschreiben „Kuschel"-Verhalten von Malacoctenus.

Die zwei Holocentridenarten, nachts aktive Fische, stehen tagsüber so ruhig zwischen den Tentakeln einer geschützt stehenden Anemone, wie sie das in einem für sie normalen Tagesversteck unter einem Stein oder in einer Spalte ohne Anemone zu tun pflegen. In einer frei stehenden Anemone wurden sie nicht beobachtet.

Der Cynoglosside ist die einzige Art aus der
Liste, die nicht mit Sicherheit zwischen den Tentakeln wahrgenommen wurde. Er kam aus einem ausgedehnten Anemonenrasen nach Anwendung eines Atemgiftes zum Vorschein, wo er sich wahrscheinlich im Sand unter der Anemone eingegraben hatte.

Apogoniden sind nachts aktiv. Sie ziehen sich tagsüber wie die Holocentriden unter Steine und in Spalten zurück aber auch zwischen die Tentakeln von freistehenden Anemonen, wo sie ruhig dicht bei einander stehen. Sie verlassen diese Plätze bei Anbruch der Dämmerung zur Nahrungssuche. Nur ein Apogon spec. mit Brut im Maul wurde auch nachts zwischen den Tentakeln angetroffen.

Einige Male wurde ein dort häufiger Zackenbarsch (Cephalopholis fulva, Serranidae) als Jungfisch beobachtet, der ruhig durch viele Tentakeln schwamm auf dem Weg von einer Stelle, wo er auf Beute gelauert hatte, zu einer anderen Lauerstelle. Die Tentakeln blieben entspannt dabei.

Junge Chaetodontiden (ca. $1 \mathrm{~cm}$ ) wurden nur selten beobachtet und wenn dann in der Nähe von oder zwischen Tentakeln: zwei Schmetterling- 


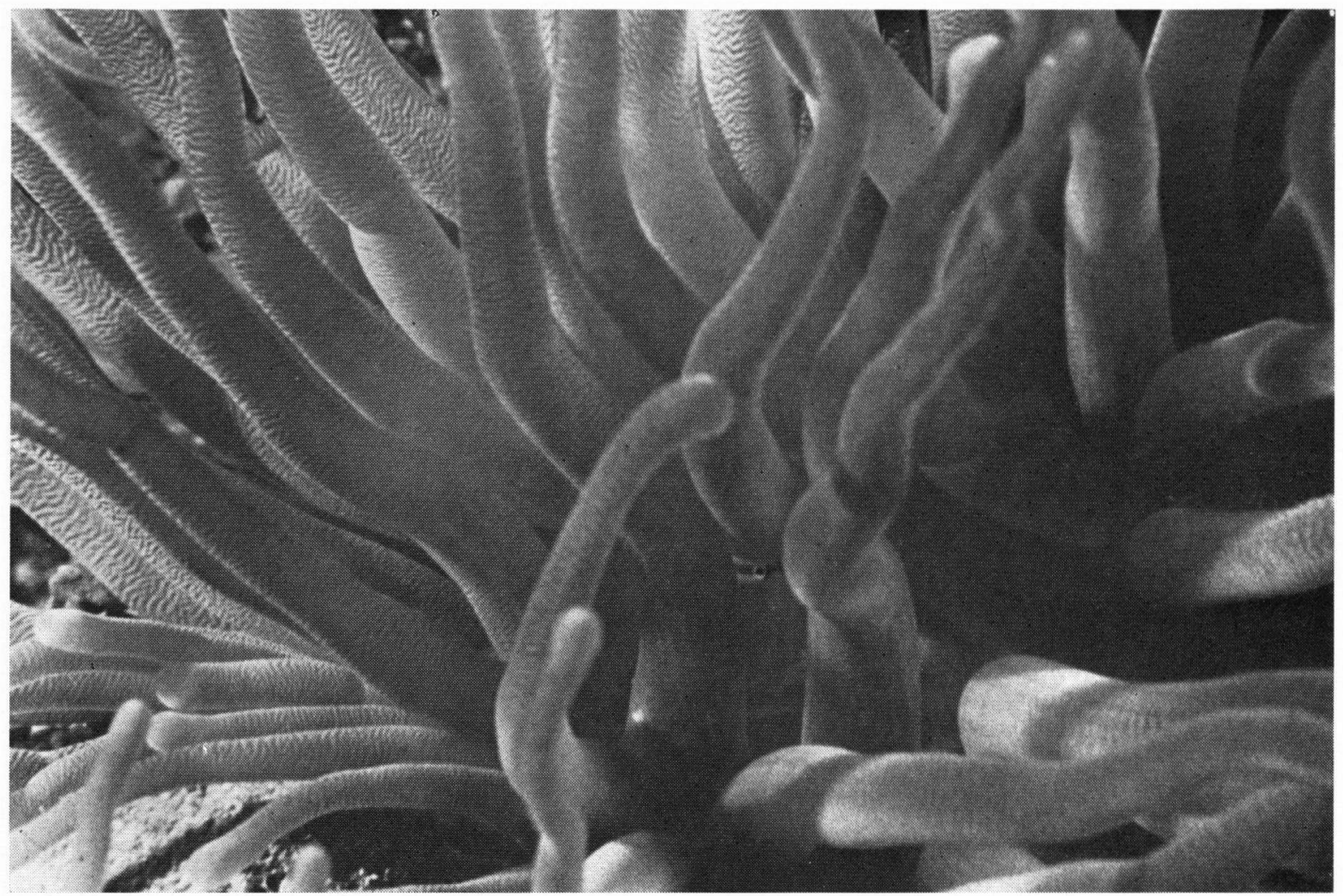

Abb. 2. Junge von Thalassoma bifasciatum sind am häufigsten in Anemonen anzutreffen.

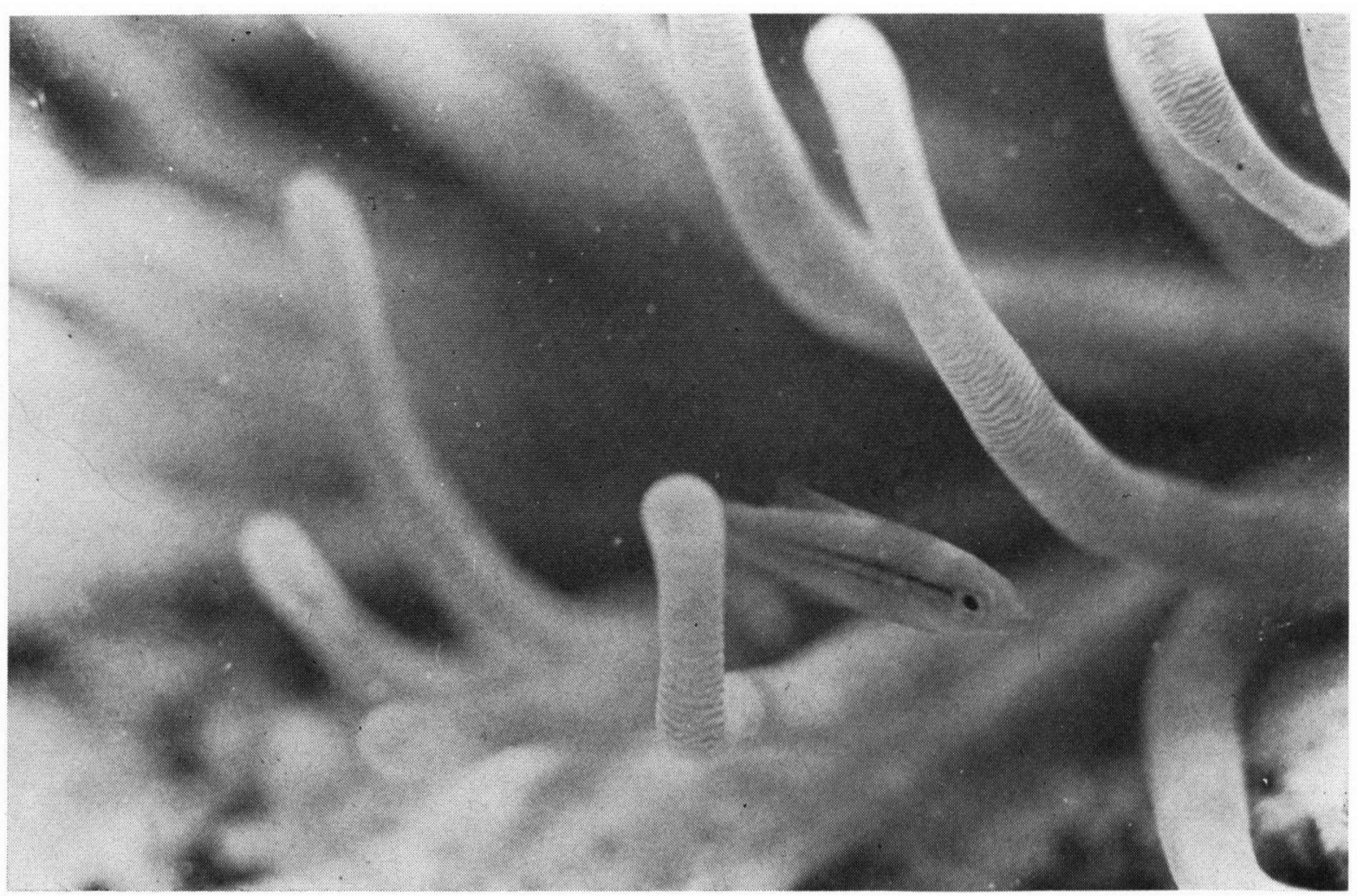

Abb. 3. Halichoeres garnoti ist ein anderer Lippfisch, der in Anemonen Schutz findet. 
fischarten in den Aussenbezirken, ein Engelfisch tief zwischen den Tentakeln. Dort verhielten die Fische sich ruhig.

Zwei Pomacentridenarten waren häufige Anemonennachbarn. Oft sah man grosse Schwärme Jungf ische von Chromis multilineata und Eupomacentrus partitus über einzelnen Anemonen oder Anemonengruppen stehen und zugleich zwischen die Tentakeln tauchen. Nachts schliefen die Tiere in Deckung am Boden, oft zwischen den Tentakeln. Auch adulte Eupomacentrus partitus sah man gelegentlich tagsüber und auch nachts bei und zwischen den Tentakeln.

Wenn man gelegentlich junge Cirrhitiden sah, dann war das in der Nähe von Anemonen. Sie sassen ruhig am Boden, stützten sich auf ihre Brustflossen und warteten auf vorbei schwimmende Beutetiere; wenn sie dabei von Anemonententakeln umspült wurden, blieben sie ruhig sitzen.

Sechs Lippfischarten waren vor allem als Jungfische bei und in Anemonen zu sehen. Der häufigste Anemonenfisch überhaupt war Thalassoma bifasciatum. Diese Art führt in Schwärmen ein unstetes Leben. Bis 10 Individuen aus einem Schwarm Jungfische tauchen immer wieder hier und da tief in eine Anemone; ganz kleine $(1 \mathrm{~cm})$ bleiben länger bei einer Anemone. Dies gilt auch für die anderen fünf Arten (siehe Liste). Keine Art scheint an eine bestimmte Anemone gebunden zu sein. Doch wurde einige Male festgestellt, dass junge Thalassoma bifasciatum, von uns von einer Anemone verjagt, aus Abständen bis $5 \mathrm{~m}$ zurückkehrten. Ungestört entfernten sich einige Fische bis $1 \mathrm{~m}$ weit.

Für junge Papagaifische gilt das Gleiche. Bei etwa 1-2 cm Länge findet man sie einzeln oder zu zweit und dritt unregelmässig in Anemonen. Werden sie grösser, dann schliessen sie sich zu kleinen Schwärmen zusammen, die durch das Riff streunen, aber mit Anemonen nicht mehr in Berührung kommen.

Die vier Clinidenarten sind die einzigen, die als Jungfische und Erwachsene ihren regelmässigen Aufenthaltsort in Anemonen haben (ausser $\mathrm{He}$ miemblemaria wahrscheinlich). Sie sitzen ruhig auf und in den Tentakeln. Ob diese Arten dieselben wie die von Hanlon \& Kaufman (1976) erwähnten Arten sind, ist noch nicht untersucht.
Die zwei Gobiidenarten sitzen auf dem Sand meist in der Nähe von Steinen und anderer Dekkung. Haften Anemonen an diesen Steinen, dann sitzen sie auch unter und manchmal auf den Tentakeln. Die Nachbarschaft scheint aber zufällig zu sein. Bei der einen Art (Coryphopterus glaucofraenum) beobachteten Hanlon \& Kaufman (1976) dasselbe.

Junge Skorpionfische wurden nur in und unter Anemonen gesehen. Über Tag sind sie so gut wie unsichtbar; erst bei Anbruch der Dämmerung kommen sie zum Vorschein und liegen, fast unsichtbar, unter und zwischen den Tentakeln.

Fast alle in der Liste genannten Arten wurden durch einen Störreiz dazu gebracht, näher zu einer Anemone und zwischen die Tentakeln zu schwimmen. So ein Störreiz konnte ein sich nähernder grösserer Fisch sein, ein grosser Schatten oder die Hand des Beobachters. Die Fische waren meist agressive Artgenossen, doch wurde auch einige Male beobachtet, wie Anemonenfische vor einem potentiellen Fressfeind in die Tentakeln auswichen. In diesem Verhalten die Bedeutung des Zusammenlebens von Fischen und Anemonen zu sehen, liegt vor der Hand. Wenn die Toleranz der Anemonen für Fische jedoch so allgemein scheint, dass auch Raubfische ungenesselt durch die Tentakeln schwimmen können, so wie der für alle anderen Anemonenfische gefährliche Cephalopholis fulva, dann wird es fraglich, ob die Anemone überhaupt Schutz bietet. Auffälliges „Verstecken" sah man nur bei Apogon maculatus, Apogon spec., Centropyge argi, Chromis multilineata, Eupomacentrus partitus und Eupomacentrus leucostictus. Bei weiteren Versuchen, Fische in die Tentakeln zu scheuchen, ergab sich, dass einige Arten, die in der gleichen Umgebung vorkamen und oft mit Anemonenfischen schwärmten, nicht zwischen die Tentakeln schwammen. Das waren Junge von zwei Hypoplectrus-Arten, von Haemulon flavolineatum, von Acantburus babianus und Acantburus chirurgus und von Canthigaster rostrata. Diese Arten flüchteten immer, auch nach vielfältigen Versuchen, von den Tentakeln weg. Zwar war keine dieser Arten ein potentieller Fressfeind von Anemonenfischen, doch war damit eine gewisse Artspezifizität der Anemonen erwiesen, und das ist eine Voraussetzung dafür, dass gewisse Fischarten 


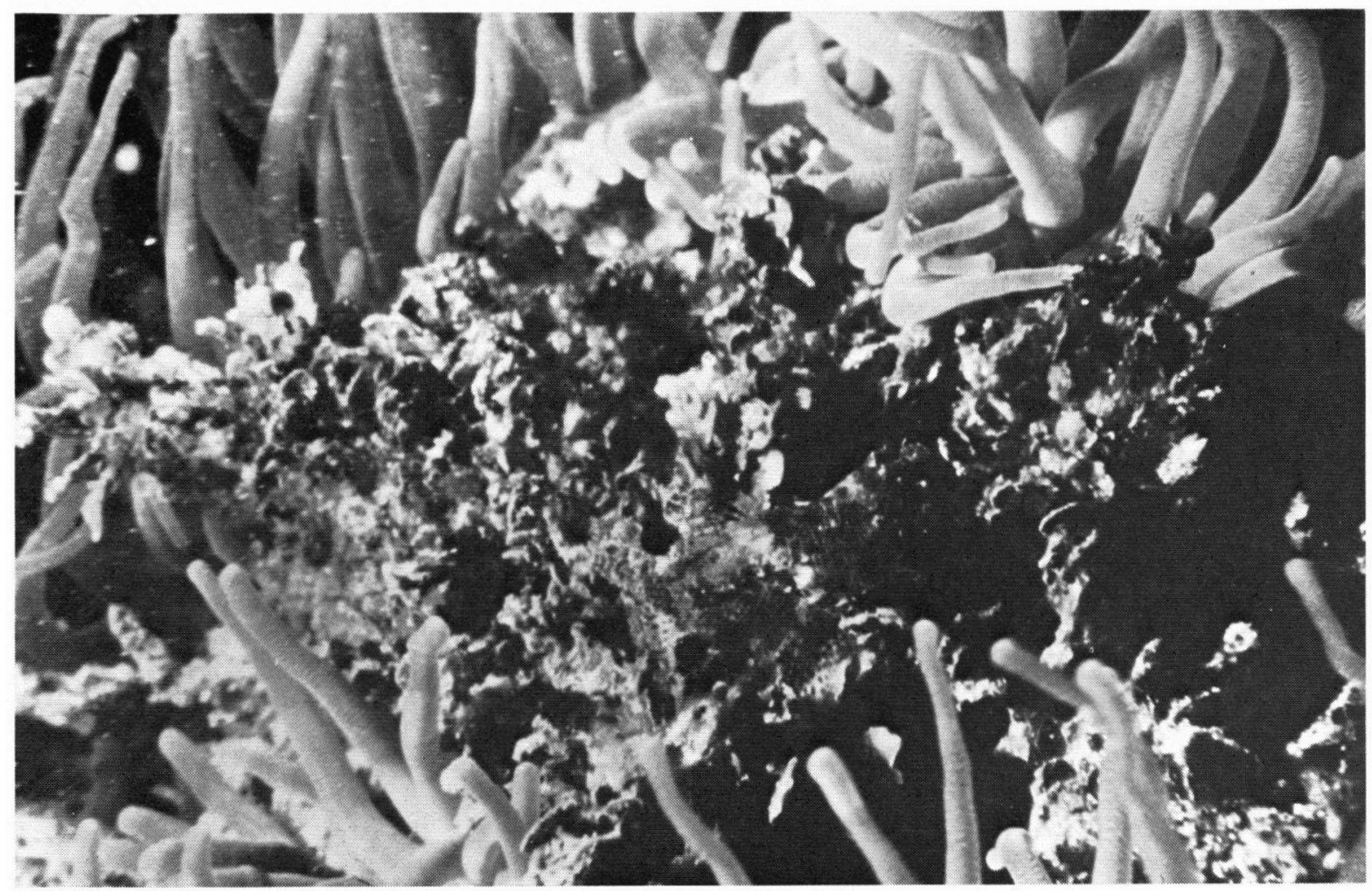

Abb. 4. Scorpaena plumieri Jungfische kommen nachts aus ihren Verstecken und sind auch dann fast unsichtbar.

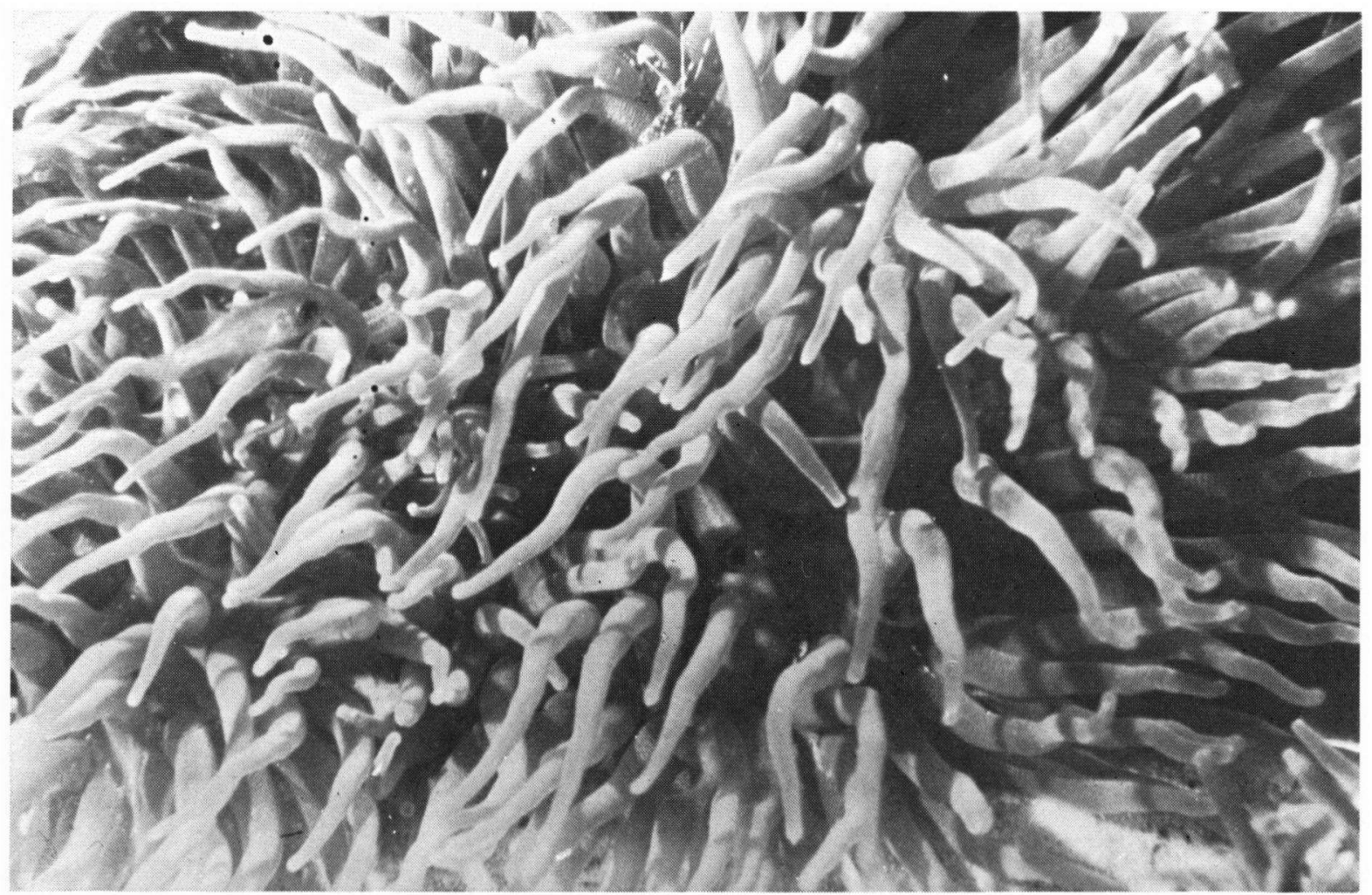

Abb. 5. Apogon spec. versteckt sich tagsüber in Tentakeln. 
in Anemonen Schutz finden. Es wurden nun die folgenden Experimente ausgeführt mit dem Ziel, die Reaktion der Anemonen auf Fische genauer zu untersuchen.

\section{EXPERIMENTE}

Es wurden Experimente mit lebenden und toten Fischen und mit Fremdkörpern durchgeführt. Die lebenden wurden mit kleinen Netzen gefangen, und unter Wasser in einen etwa $30 \mathrm{~cm}$ langen Plastiksack gelassen, der dann über eine Anemone gestülpt wurde. Dann wurde das Wasser aus dem Plastiksack langsam herausgedrückt, was die Fische im Laufe von etwa 1 min. an die Tentakeln brachte. Wenn die Fische nicht gleich zu Anfang ruhig in die Tentakeln schwammen, wurden sie bei zunehmendem Platzmangel immer unruhiger bis sie panikartig mit den Tentakeln in Berührung kamen.

Erwartungsgemäss wurden kleine Haemulon, die immer Tentakeln meiden, sogleich gefangen, gelähmt und verschlungen. Bei Anemonenfischen gab es verschiedene Empfindlichkeiten. Gelegentliche Anemonengäste, wie Gobies, klebten fast so schnell wie Haemulon und wurden gefressen. Schleimfische flüchteten sofort in die Tentakeln. Wenn sie von uns wieder herausgejagt wurden, war es schwierig, sie ein zweites Mal hineinzujagen. Beim dritten und vierten Mal sah man deutlich die Ursache dafür: der Fisch klebte fest und wurde aufgefressen. Auch Thalassoma, neben Schleimfischen die häufigsten Anemonenfische, versteckten sich gut in den Tentakeln, und man musste eine Anemone drücken und zausen, bis so ein Fisch festklebte.

Nach wenigen Experimenten wurde deutlich, dass einige Anemonenfischarten eine gewisse Immunität gegen Anemonen haben; also vielleicht auch in Anemonen einen gewissen Schutz vor Fischfeinden finden können.

Ob Anemonenfische mehr durch ihr Schwimmverhalten oder mehr durch einen besonderen Schleim vor den Anemonen geschützt sind, liess sich damit nicht beantworten. $\mathrm{Da}$ ruhig in die Tentakeln schwimmende Anemonenfische besser geschützt sind als panikartig flüchtende, scheint das Verhalten eine wichtige Rolle zu spielen, v.a. bei Thalassoma und Malacoctenus. Klebt der Fisch, weil er aus Angst vor uns zappelt, oder weil wir die Anemone stören oder beides? Die Experimente waren schwierig zu standardisieren und wurden deshalb unterbrochen. Der Versuch hatte den Nachteil, dass die Fische verschieden schnell in die Anemone schwammen und fast immer schneller als freischwimmende Fische. Auf diese Weise kann ein möglicher Reiz eines Fisches auf die Tentakeln nicht mit der natürlichen Situation verglichen werden. Das war auch beim hiernach beschriebenen Versuch nicht möglich, doch waren die Berührungsreize für die Tentakeln immer etwa gleich stark und sicher meist schwächer als von lebenden Fischen.

Beim 2. Experiment wurden verschiedene Anemonenfischarten (siehe Liste) mit dem Atemgift Rotenon betäubt. Wenn sie mit langsamen Bewegungen halb betäubt aus ihren Versteckplätzen kamen, liessen wir sie in ein Marmeladenglas treiben. Aus diesem Glas liessen wir die bewegungslosen und schlaffen Fische sogleich langsam auf eine Anemone fallen oder fassten den Fisch am Schwanz mit einer Pinzette und liessen den Kopf zuerst die Anemone berühren. Die Schleimhaut der Fische wurde hierbei so gut wie sicher nicht beschädigt. Kontrollversuche mit Fleischstückchen, mit und ohne Rotenon-Behandlung, liessen keinen Einfluss dieses Stoffes auf die Reaktion der Anemonen erkennen. Das „Verhalten" der Fische bei diesem Experiment war praktisch gleich. Aber nur Thalassoma wurde nicht von der Anemone festgehalten. Er könnte durch seinen Schleim vor der Anemone geschützt sein. Hanlon \& Kaufmann (1976) experimentierten mit betäubten Malacoctenus (4 Arten) und Haemulon (1 Art). Sie geben nicht an, ob sie die Schleimhaut der Fische intakt liessen. Ein Malacoctenus wurde dabei getötet aber von der Anemone nicht gefressen. Vielleicht ist auch er durch seinen Schleim geschützt.

Wahrscheinlich spielen beide, Schleim und Verhalten eine wichtige Rolle. $\mathrm{Zu}$ dieser Schlussfolgerung kam auch Eibl (1964) in seiner Diskussion der Schleim vs. Verhalten-Kontroverse von Davenport \& Norris (1958) und Graefe (1964) über die Anemonenfische der Gattung Amphiprion.

Bei einigen Versuchen befanden sich in der Anemone Fische, während sie einen Experimentierfisch bekamen. Apogon schienen in einer fressenden 

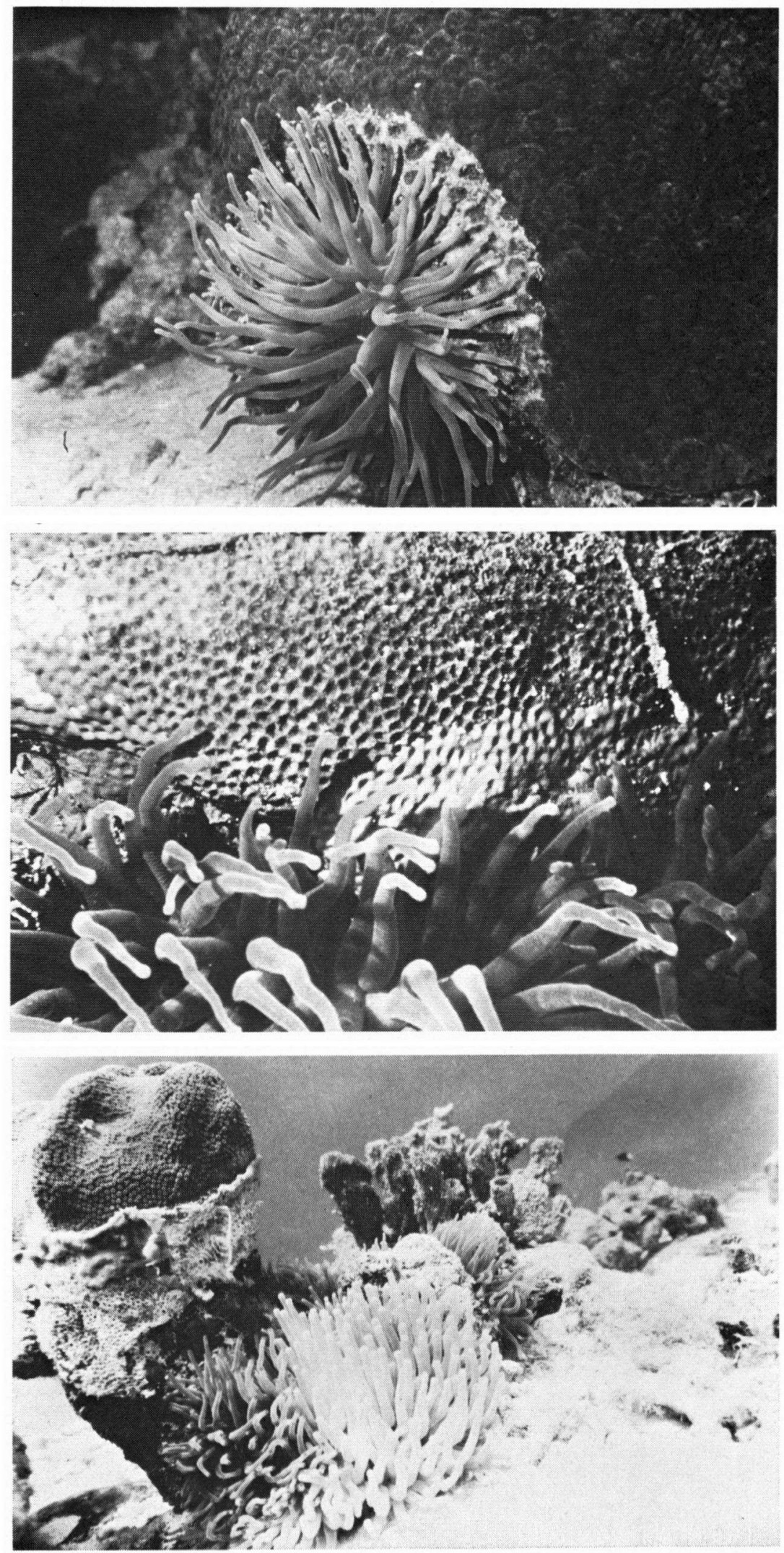

Abb. 6-8. Drei Stadien der Anemonen-Korallen Antibiose. 
Anemone nicht mehr so sicher, sie wurden "gejuckt", Thalassoma blieben völlig unbehelligt. Kontrollversuche (etwa 100) mit Fleischstücken, anstatt Experimentierfischen, die von den Anemonen eben so schnell gef ressen wurden, brachten jedoch ein negatives Ergebnis, sowohl Thalassoma als Apogon blieben bei diesen Versuchen unbehelligt.

Kleine Steinchen (ca. $15 \times$ ), die wir zur Kontrolle auf Tentakeln fallen liessen, klebten kurz und fielen ab. Kleine Nacktschnecken $(2-3 \mathrm{~cm})$ klebten nicht $($ ca. $15 \times)$ und rollten passiv von den entspannt bleibenden Tentakeln. Schwammstücke klebten fest und wurden verschlungen. Daraus lässt sich schliessen, dass die Schnecken über einen Schleim verfügen, der das Auslösen der Nesselkapseln hemmt.

\section{DISKUSSION}

Hanlon \& Kaufman (1976) unterscheiden zwei Typen von Anemonenfischen: Bodenfische ruhen auf und zwischen den Tentakeln, freischwimmende Fische hingegen vermeiden direkten Kontakt. Diese Einteilung lässt sich mit dem vorliegenden Material nicht machen.

Aus der Tabelle wird deutlich, dass sich ausser den Schleimfischen und ev. der Hundszunge - praktisch nur Jungfische in den Tentakeln von Condylactis aufhalten. Im Schutz dieser Jungf ische vor Fressfeinden die biologische Bedeutung der Vergesellschaftung zu sehen ist naheliegend.

In ungestörten Riffen ist der Fischartenreichtum grösser als in halbzerstörten Riffen. Die Anzahl der Individuen pro Art scheint in ungestörten Riffen jedoch kleiner; dies schien am deutlichsten bei den genannten Labriden und besonders bei Thalassoma bifasciatum der Fall zu sein. Diese Art ist der häufigste Anemonenfisch. Vielleicht gedeiht er in gestörten Riffen Curaçaos besonders gut, weil er Schmutzwasser besser verträgt als andere Arten oder weil Jungfische dieser Art in diesen Riffen besonders gut geschützt sind durch die vielen Anemonen.
Vielleicht sind die Anemonen in halbtoten Riffen so zahlreich, weil es so viel verfügbares Substrat gibt. In den untersuchten unberührten Riffen sind sie schwer $\mathrm{zu}$ finden und bleiben klein. Praktisch jeder Quadratzentimeter ist von Steinund Lederkorallen besetzt. Es sieht so aus als ob es dort keinen Platz für Condylactis gibt.

Burkholder (1973) nennt in seinem zusammenfassenden Artikel über Antibiosis viele andere Coelenteraten, die einen wachstumshemmenden Einfluss auf Organismen in ihrer Umgebung ausüben. Wenn so eine Antibiosis von Stein- und Lederkorallen gegen Condylactis besteht, dann wirkt sie in gestörten Riffen auf jeden Fall entgegengesetzt. Die Tentakeln von Condylactis töten nämlich alle Stein-Korallenpolypen in ihrer Reichweite. Auf den kahlen Skeletten siedeln sich Schwämme und Feuerkorallen an, die z.T. offenbar den Korallenstock weiter erodieren. Bis zu völlig ausgehöhlten und umgestürzten Korallenstöcken lassen sich alle Übergänge finden. Condylactis kann so aktiv an der Weiterzerstörung des Riffs mitwirken (Abb. 6-8), wenn es erst einmal aus dem Gleichgewicht geraten ist.

Welche andere ökologische Nischen die genannten Fische benutzen, wenn sie nicht in Anemonen gehen, ist noch unbekannt. Nach Allen (1975) sind die Verbreitung und Dichtheit von Amphiprion-Anemonen wichtige Faktoren, die die Verbreitung von Amphiprion beeinflussen.

Zwar meiden die bisher bekannten indopazifischen Anemonenfische - ausser Amphiprion nach Allen (1975) direkte Berührung mit den Tentakeln von Anemonen. Bei der Hälfte dieser Arten handelt es sich aber um kleine Arten und bei den anderen um Jungfische. Das stimmt auffällig überein mit den karibischen Befunden. $\mathrm{Da}$ es sich bei den indopazifischen Anemonenfischen - ausser Amphiprion - fast nur um Gelegenheitsbeobachtungen handelt, wird man bei näherem Hinsehen wahrscheinlich eine ebensolche Vergesellschaftung von Jungfischen mit Anemonen finden. Schlichter (1968) machte damit bereits einen Anfang.

\section{LITERATURVERZEICHNIS}

Abel, E. F., 1960. Liaison facultative d'un Poisson (Gobius bucchichii Steindachner) et d'une anemone (Anemonia sulcata Penn.) en Méditerranée. Vie Milieu, 11: 517-531. AlleN, G. R., 1975. The anemone fishes, their classification 
and biology, 2nd ed.: 1-352. (T.F.H. Publications Inc. Ltd., Hong Kong.)

Böhlke, J. E. \& Ch. G. Chaplin, 1968. Fishes of the Bahamas and adjacent tropical waters: 1-771. (Harrowood Books, Valley Forge, Pa.)

Burkholder, P. R., 1973. The ecology of marine antibiotics and coral reefs. In: O. A. JoNEs \& R. ENDEAN (eds.) Biology and geology of coral reefs: 117-182. (Academic Press, New York, London.)

Chaplin, Ch. G., 1972. Fishwatchers guide: 1-641. (Harrowood Books, Valley Forge, Pa.)

Colin, P. L. \& J. B. Heiser, 1973. Associations of two species of cardinal fishes (Apogonidae: Pisces) with sea anemones. Bull. mar. Sci., 26 (2): 225-232.

DAvenport, D. \& K. S. Norris, 1958. Observations on the symbiosis of the sea anemone Stoichactis and the pomacentrid fish, Amphiprion percula. Biol. Bull., 115 (3): 397-410.

Elbl-EibesfeldT, I., 1964. Im Reich der tausend Atolle: 1-195. (Piper, München.)
Graefe, G., 1964. Zur Anemonen-Fish-Symbiose, nach Freilanduntersuchungen bei Eilat/Rotes Meer. Z. Tierpsychol., 21: 468-485.

Hanlon, R. T. \& L. Kaufman, 1976. Associations of seven West Indian reef fishes with sea anemones. Bull. mar. Sci., 26 (2): 225-232.

Hannau, H. W. \& B. H. Mock, 1974. Beneath the seas of the West Indies: 1-104. (Argos Inc., Miami, Fla.)

Mariscal, R., 1966. The symbiosis between tropical sea anemones and fishes: a review. In: R. I. BowmaN (ed.), The Galapagos: 157-171 (Berkeley Univ. Press.)

PaX, F., 1910. Studien an westindischen Aktinien. Zool. Jahrb., Suppl. 11: 157-330.

SCHLICHTER, D., 1968. Zusammenleben von Riffanemonen und Anemonenfischen. Z. Tierpsychol., 25: 933-954.

SMITH, W. L., 1973. Labrisomus kalichera (Clinidae) in Condylactis gigantea. Copeia, 1973 (3): 597-598.

Stevenson, R., 1963. Behavior of the pomacentrid reeffish Dascyllus albisella Gill in relation to the sea anemone Marcantia cookei. Copeia, 1963 (4): 612-614. 